\title{
Hybridizing the hybrid procedure
}

\author{
Sanjiv K. Gandhi, MD \\ From the Division of Pediatric Cardiothoracic Surgery, British Columbia Children's Hospital, Vancouver, British \\ Columbia, Canada. \\ Disclosures: Author has nothing to disclose with regard to commercial support. \\ Received for publication Oct 17, 2015; accepted for publication Oct 19, 2015; available ahead of print Nov 18, \\ 2015. \\ Address for reprints: Sanjiv K. Gandhi, MD, Division of Pediatric Cardiothoracic Surgery, British Columbia \\ Children's Hospital, 4480 Oak St, Suite AB307, Vancouver, British Columbia, Canada V7V 1Y8 (E-mail: \\ sgandhi@cw.bc.ca). \\ J Thorac Cardiovasc Surg 2016;151:430-1 \\ $0022-5223 / \$ 36.00$ \\ Copyright (c) 2016 by The American Association for Thoracic Surgery \\ http://dx.doi.org/10.1016/j.jtcvs.2015.10.056
}

In their article in this issue of the Journal, Galantowicz and Yates review their extensive experience with the comprehensive stage 2 (CS2) procedure after initial hybrid stage 1 palliation in patients with hypoplastic left heart syndrome. The article details 2 groups of patients, divided by time eras; patients in the second group were managed according to an intentional series of modifications of institutional protocols designed to attempt to improve mortality. This is an important report and adds to our knowledge about managing this complex group of children.

It is clear from this analysis that, as with most surgical procedures, hybrid stage 1 palliation and the subsequent CS2 procedure require careful patient selection to optimize outcomes. Careful scrutiny of the "preprotocol" group of patients revealed pulmonary artery (PA) thrombosis to be the most common mode of death, with an urgent indication for surgery and young age also being significant factors contributing to mortality. Care modifications in the "postprotocol" group included no emergency procedures or procedures in children younger than 3 months of age, selective use of a Blalock-Taussig shunt if the superior vena cava or PAs were deemed too small, a completion angiogram with a low threshold for intraoperative stenting, and postoperative anticoagulation for 6 weeks. There were 6 patients in the postprotocol group deemed ineligible for the CS2 procedure; these underwent either an interim arch procedure or a Blalock-Taussig shunt instead of a Glenn during part 1 of stage 2 . Although the sizes of the superior vena cava and PAs were assessed intraoperatively, it is not entirely clear how this assessment was objectively accomplished and thus how small was considered too small. It seems that this staged approach to stage 2, although perhaps necessary because of anatomic constraints, defeats the purpose of the initial hybrid stage 1. Perhaps if a mechanism existed to predict which patients would require an interim procedure, these children could be treated with the standard Norwood operation as a neonate. In this series, approximately $10 \%$ of the postprotocol patients were not included in the hybrid stage 1 to CS2 pathway but were

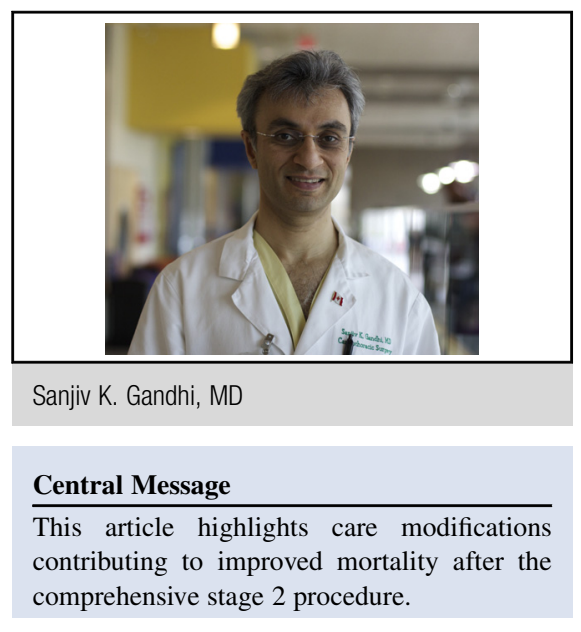

See Article page 424

rather scheduled to undergo 3 major procedures before their eventual Fontan procedure.

With respect to immediate postoperative outcomes, the primary outcome measure in the article is operative mortality. Galantowicz and Yates focused on the complications of extracorporeal membrane oxygenation, PA thrombosis, and bleeding. Additional morbidities for those patients who did not survive are provided in Table 4 of their article. It is imperative there be some assessment of the morbidity of the CS2 procedure for the entire cohort of patients. What were the ventilation times for these kids? How often were sternums left open? What were the postoperative inotropic requirements? What were the rates of acute kidney injury? What were the rates of chylothorax, phrenic injury, and recurrent laryngeal nerve injury? What were the incidences of infectious complications? What was the status of children at discharge-for example, were most kids eating on their own, or were feeding tubes frequently used? What were the rates of early readmission? Was there a difference in these morbidities between the 2 groups of patients? It would be instructive to learn why the intensive care unit and hospital of stays were longer for the postprotocol patients, who seemed to be more carefully selected to eliminate some of the higher risk subset of kids.

The larger question in this patient population relates to longer-term outcomes, that is, Fontan candidacy, which of course is the ultimate goal of the stage 1 and 2 procedures, whatever those procedures may be. What will be the future effects on children receiving PA stents, such as is demonstrated in the article's Figure 4? This stent seems to 
occupy most of the left PA and most surely will affect future left PA growth and perhaps even Fontan candidacy, which is the end point for all of these patients. One of the primary determinants of a good Fontan circulation is good PAs. By Galantowicz and Yates's own admission, the hybrid pathway leads to significant PA issues, starting from the initial hybrid palliation, because of several factors outlined in their discussion section. "A disrupted endovascular bed" could be a bad starting place for an ultimate Fontan circulation. Even if the rate of successful Fontan completion is the same between the hybrid and traditional Norwood groups, the presence of a stented PA will inevitably lead to even more PA interventions and perhaps more Fontan-related morbidity in the future.

The theoretic benefits of the hybrid procedure relate to the avoidance of cardiopulmonary bypass and circulatory arrest in the neonatal period. Galantowicz and Yates should be commended for pushing the hybrid pathway approach to these complex children, but a single overarching question remains: Is the hybrid strategy better than the standard Norwood single-ventricle pathway? Although this study cannot definitively answer that question, it adds to the growing body of literature trying to find the appropriate indications for this mode of therapy. ${ }^{1-4}$

\section{References}

1. Baba K, Kotani Y, Chetan D, Chaturvedi RR, Lee KJ, Benson LN, et al Hybrid versus Norwood strategies for single-ventricle palliation. Circulation. 2012;126(11 Suppl 1):S123-31.

2. Davies RR, Radtke WA, Klenk D, Pizarro C. Bilateral pulmonary arterial banding results in an increased need for subsequent pulmonary artery interventions. J Thorac Cardiovasc Surg. 2014;147:706-12.

3. Dave H, Rosser B, Knirsch W, Hübler M, Prêtre R, Kretschmar O. Hybrid approach for hypoplastic left heart syndrome and its variants: the fate of the pulmonary arteries. Eur J Cardiothorac Surg. 2014;46:14-9.

4. Rahkonen O, Chaturvedi RR, Benson L, Honjo O, Caldarone CA, Lee KJ Pulmonary artery stenosis in hybrid single-ventricle palliation: high incidence of left pulmonary artery intervention. J Thorac Cardiovasc Surg. 2015;149: 1102-10.e2. 